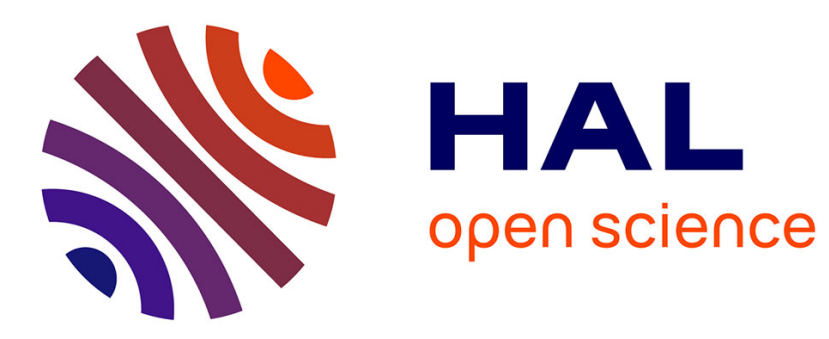

\title{
Constructing Weighted Argumentation Framework with Cognitive Maps
}

\author{
Karima Sedki, Louis Bonneau de Beaufort
}

\section{To cite this version:}

Karima Sedki, Louis Bonneau de Beaufort. Constructing Weighted Argumentation Framework with Cognitive Maps. International Conference on Tools with Artificial Intelligence (ICTAI), IEEE, Nov 2015, Vietri sul Mare, Italy. 10.1109/ICTAI.2015.108 • hal-01231192

\section{HAL Id: hal-01231192 \\ https://hal.science/hal-01231192}

Submitted on 19 Nov 2015

HAL is a multi-disciplinary open access archive for the deposit and dissemination of scientific research documents, whether they are published or not. The documents may come from teaching and research institutions in France or abroad, or from public or private research centers.
L'archive ouverte pluridisciplinaire HAL, est destinée au dépôt et à la diffusion de documents scientifiques de niveau recherche, publiés ou non, émanant des établissements d'enseignement et de recherche français ou étrangers, des laboratoires publics ou privés. 


\section{Constructing Weighted Argumentation Framework with Cognitive Maps}

\author{
Karima Sedki \\ 1. LIMICS (INSERM UMRS 1142), Université Paris 13, \\ Sorbonne Paris Cité, 93017 Bobigny, France. \\ UPMC Université Paris 6, Sorbonne Universités, Paris \\ Email: karima.sedki@univ-paris13.fr
}

\author{
Louis Bonneau de Beaufort \\ 1. AGROCAMPUS OUEST, \\ UMR6074 IRISA, F-35042 Rennes, France \\ 2. Université européenne de Bretagne, France \\ Email: louis.bonneau@agrocampus-ouest.fr
}

\begin{abstract}
Cognitive map is a qualitative decision model which is frequently used in social science and decision making applications. This model allows to easily organize individuals' judgments, thinking or beliefs about a given problem in a graphical representation containing different concepts and influences between them. However, reasoning on this model presents some limits and remains a difficult task. For example, cognitive maps do not model uncertainty within the variables, and only deductive reasoning (predicting an effect given a cause) is possible. In this paper, we show how to translate the knowledge represented in cognitive maps in the form of arguments and attack relations among them. In particular, given a decision problem, a cognitive map was first built by eliciting knowledge from experts and then transforming it in a weighted argumentation framework (WAF for short) for ensuring efficient reasoning. Another contribution of this paper concerns enriching the WAF obtained from a given cognitive map for dealing with dynamics through the consideration of a varying set of observations.
\end{abstract}

Keywords-Cognitive maps, weighted argumentation framework, observations, active arguments.

\section{INTRODUCTION}

There exists many formalisms for knowledge representation and reasoning. We can cite logical ones like argumentation theory, possibilistic logic, or graphical ones like Bayesian networks, cognitive maps. However, following the used formalism, eliciting the model, or using it for reasoning (an important task in decision making) can be more or less tedious. Concerning the elicitation step, the difficulty appears namely in the used variables (factors, events, etc.), the different kinds of relationships between variables (dependencies, causality, correlation, etc.) the nature of data (uncertain, incomplete, etc.), etc. In addition, the challenge concerns the gathering of knowledge: some formalisms, like the logical ones, require a formal representation that human experts might be unable to provide. About the reasoning step which consists in obtaining new facts or conclusions from other information, or explaining a fact, the difficulty concerns namely the nature of reasoning (diagnosis, prediction, under uncertainty and missing information, etc.).

Transformations from a given formalism into another is an idea that many researchers have followed. For example, transforming probability measures into possibilistic ones is proposed in [15], [1]. Such transformations can be helpful in many situations such as reusing the existing tools (such as algorithms and software) developed in one setting, instead of developing again tools for the new setting.

In this paper, we consider two formalisms. The first one is cognitive maps (CMs for short) that represent influences between concepts in an intuitive and easy to interpret representation [8], [4]. The second one is weighted argumentation framework [7] which consists of constructing arguments and counter-arguments for, or against, some statements. In WAF, each attack among arguments is associated with a numerical value indicating the strength of that attack. More precisely, we are mainly interested in transforming cognitive maps into weighted argumentation frameworks. This is motivated by the fact that we would like to preserve what's good in cognitive maps and overcome some of their limitations: even if cognitive maps are easier to grasp and close to the way in which knowledge is represented by human beings, reasoning about the represented knowledge has some limits. The first reason of this limitation in cognitive maps is that they do not model uncertainty within the variables. In addition, they allow only limited forms of inferences. Besides, asking human experts to represent their knowledge, judgments and beliefs as structured arguments and how arguments may interact is a great challenge, and a highly time-consuming one. However, reasoning with WAF is very interesting, especially in situations of inconsistency. As shown in Section V, another contribution of this paper concerns enriching the WAF obtained from a given cognitive map for dealing with dynamics through the consideration of a varying set of observations. For example, when dealing with a fishing activity problem, having an evidence or an observation about an increase in the number of fishers, only some arguments be active and other do not. Some works dealing with dynamics in argumentation theory can be found in [12], [3], [9], [13].

In cognitive maps, each relation between two concepts is associated with a numerical value (positive or negative) indicating the strength of the influence between them. In weighted argumentation frameworks, weights are assigned to 
each attack which represents the strength of the attacking argument on its attacker. Namely, the higher the weight, the stronger is the attack. Thus, the link between the cognitive map and weighted argumentation framework can be naturally established.

The rest of the paper is organized as follows: In section II, we present some important issues related to cognitive maps. In Section III, we give some elements about Dung argumentation framework and weighted argumentation framework. In Section IV, we present the translation of a cognitive map into a weighted argumentation framework. Section V presents the reasoning process in the obtained WAF. Section VI concludes the paper.

\section{Cognitive Maps}

A cognitive map is a directed graph that represents influences between concepts. It expresses individuals' judgments, thinking or beliefs about a given problem [8], [4]. In this paper, we use the formalism presented in [14] where influence relations are not necessary symmetric, contrary to the original formalism.

Definition 1 (Cognitive map): A cognitive map is a directed graph $C M=\langle\mathcal{C}, \mathcal{I}, \mathrm{V}$, val $\rangle$ where:

- $\mathcal{C}$ is a set of concepts that are the nodes of the graph,

- $\mathcal{I}$ is a set of arcs that represent influences between concepts. If there is an influence from concept $A$ towards a concept $B$, then $A$ is called a causal concept and $B$ is called an effect concept.

- $V$ is a set of values representing the strength of the influence between concepts ${ }^{1}$.

- val: $\mathcal{I} \rightarrow V$ is a function that assigns two values to each influence, denoted by $\left[v, v^{\prime}\right]$.

In cognitive maps, there are two types of influences: positive and negative. The positive means that the causal concept stimulates increasing of the effect concept. The negative influence means that the causal concept stimulates decreasing of the effect concept. Each influence is assigned with two values represented by $\left[v, v^{\prime}\right]$ where $v$ represents the influence degree on the effect concept when the causal concept decreases. $v^{\prime}$ represents the influence degree on the effect concept when the causal concept increases. Formally, the state increasing (resp. decreasing) of a given concept $c_{1}$ is represented by $c_{1}^{+}$(resp. $c_{1}^{-}$). Thus, we use the notation State $\left(c_{1}\right)=\left\{c_{1}^{-}, c_{1}^{+}\right\}$to represent the possible states of $c_{1}$.

Property 1: Let $C M=\langle\mathcal{C}, \mathcal{I}$, V, val $\rangle$ be a cognitive map, $c_{1}, c_{2}$ be two concepts in $\mathcal{C}, I$ in $\mathcal{I}$ represents the influence between $c_{1}, c_{2}$ and $\operatorname{val}(I)=\left[v, v^{\prime}\right]$. Then, it holds that following the value $v$ (resp. $v^{\prime}$ ), we have:

\footnotetext{
${ }^{1}$ We let $V$ denote the natural numbers defined on the interval $[-3,+3]$. If the value is equal to -1 (resp. +1 ), then the strength of the influence is low and negative (resp. positive), medium for $(-2$ or +2$)$, High for $(-3,+3)$, and Null for (0). These values are used for the drawing step of $C M s$ since they are easier, more intuitive, for elicitation.
}

- If $\operatorname{State}\left(c_{1}\right)=c_{1}^{-}$then $\operatorname{State}\left(c_{2}\right)$ is either $c_{2}^{-}$if $\mathrm{v}<0$ or $c_{2}^{+}$if $v \geq 0$ ( $c_{2}^{-}$and $c_{2}^{+}$are mutually exclusive).

- If $\operatorname{State}\left(c_{1}\right)=c_{1}^{+}$then $\operatorname{State}\left(c_{2}\right)$ is either $c_{2}^{-}$if $v^{\prime}<0$ or $c_{2}^{+}$if $v^{\prime} \geq 0\left(c_{2}^{-}\right.$and $c_{2}^{+}$are mutually exclusive).

A congnitive map allows only deductive reasoning (predicting an effect given a cause). Thus, we can get responses about the effects of a given cause but we can't give explanations why effects are produced. However, if there are circular relations that can represent dynamic relations between variables over time, then deductive reasoning is not satisfactory. For example, it is possible to have a circular relation between the fishing activity variable and the fish stock variable.

Example 1: The cognitive map of Figure 1 represents influences between five concepts related to a problem of fishing activity. In order to construct the CM, interviews are conducted with fishermen. These latter are asked to identify the concepts that might influence negatively or positively the fish stock. Note that the cognitive map of Figure 1 represents only a part of the $C M$ constructed by fishermen.

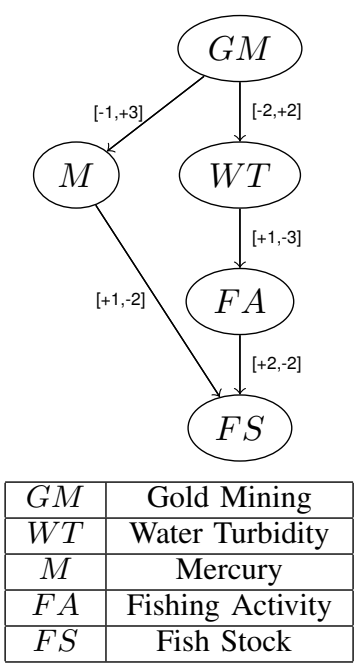

Figure 1. A cognitive map defined on the value set $[-3,+3]$

The influence between the concept $G M$ and the concept $W T$ is assigned with the values $[-2,+2]$. It means that decreasing of gold mining (i.e. $G M^{-}$) will cause a medium decrease $(-2)$ of water turbidity (i.e. $W T^{-}$) and increasing the $G M$ (i.e. $G M^{+}$) will cause a medium increase $(+2)$ of $W T$ (i.e. $W T^{+}$). The influence between the concept $W T$ and the concept $F A$ means that a decrease of $W T$ causes a small increase $(+1)$ of $F A$. An increase of $W T$ causes a high decrease $(-3)$ of $F A$.

\section{Argumentation Framework: Preliminaries}

This section briefly recalls Dung's abstract argumentation framework and weighted argumentation framework.

\section{A. Dung's argumentation framework}

An argumentation framework [6] is defined on a set of arguments and a set of attacks between them. An argument gives 
reasons for accepting or believing a statement, etc. An attack expresses conflicts between arguments. Each argumentation system can be represented with a directed graph where nodes are the arguments, and edges represent attacks between them.

Definition 2 (Dung's argumentation framework): An argumentation framework is a pair $A F=\langle\mathcal{A}, \mathcal{R}\rangle$ where $\mathcal{A}$ is a finite set of arguments and $\mathcal{R}$ is a binary attack relation defined on $\mathcal{A} \times \mathcal{A}$. Given two arguments $\sigma$ and $\gamma, \sigma \mathcal{R} \gamma$ (or $(\sigma, \gamma) \in \mathcal{R})$ means $\sigma$ attacks $\gamma$.

There can exist several extensions to $A F s$ : preferred, stable, grounded, etc. They are also called acceptability semantics.

Definition 3: Let $A F=\langle\mathcal{A}, \mathcal{R}\rangle$ be an argumentation framework and $\mathcal{S}$ be a subset of arguments (i.e., $\mathcal{S} \subseteq \mathcal{A}$ ):

- $\mathcal{S}$ is a conflict-free of $A F$ iff there are no arguments $\sigma$, $\gamma \in \mathcal{S}$ such that $\sigma \mathcal{R} \gamma$.

- $\sigma \in \mathcal{A}$ is acceptable with respect to $\mathcal{S}$ iff $\forall \gamma \in \mathcal{A}$ such that $\gamma \mathcal{R} \sigma, \exists \theta \in \mathcal{S}$ such that $\theta \mathcal{R} \gamma$

- $\mathcal{S}$ is an admissible extension iff it is conflict-free and each argument in $\mathcal{S}$ is acceptable with respect to $\mathcal{S}$.

- $\mathcal{S}$ is a preferred extension iff it is maximal (for set inclusion) among admissible extensions.

Other acceptability semantics (stable, grounded semantics for example) are defined in [6].

Example 2: Let us consider the argumentation framework $A F=\langle\mathcal{A}, \mathcal{R}\rangle$ given in Figure 2 where the set of arguments is $\mathcal{A}=\left\{\sigma_{1}, \sigma_{2}, \sigma_{3}\right\}$, the set of attacks is $\mathcal{R}=\left\{\left(\sigma_{1}, \sigma_{2}\right)\right.$, $\left.\left(\sigma_{2}, \sigma_{3}\right),\left(\sigma_{3}, \sigma_{2}\right)\right\}$. This framework contains three admissible sets which are $\left\{\sigma_{1}\right\},\left\{\sigma_{3}\right\},\left\{\sigma_{1}, \sigma_{3}\right\}$ and only one preferred extension which is $\left\{\sigma_{1}, \sigma_{3}\right\}$.

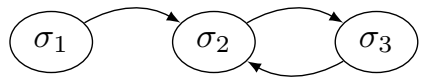

Figure 2. An example of $A F$

\section{B. Weighted argumentation framework}

In [7], the authors introduce weighted argumentation framework which is an extension of Dung argumentation framework. In WAF, a weight (a positive real number) is associated with each attack. The attack from $\sigma$ to $\sigma^{\prime}$ which is associated with a weight $w$ is considered to be stronger than the attack from $\sigma^{\prime}$ to $\sigma$ with a weight $w^{\prime}$ iff $w>w^{\prime}$.

Definition 4 (Weighted Argumentation Framework): A Weighted Argumentation Framework is a triple WAF $=\langle\mathcal{A}, \mathcal{R}, w\rangle$ where $\langle\mathcal{A}, \mathcal{R}\rangle$ is a Dung abstract argumentation framework, and $w: \mathcal{A} \rightarrow \Re^{2}$ is a function that assigns a real number to each attack.

In [7], the weight function is defined as a real value function. For the purpose of our problem (fishing activity), natural numbers are used. On the basis of attack's weights,

\footnotetext{
${ }^{2}$ We let $\Re$ denote the real numbers greater than or equal to zero.
}

an inconsistency budget $\beta$ of a set of arguments is defined in WAF. It induces the relaxation of the usual notion of conflict-free sets of arguments where some inconsistencies are tolerated in sets of arguments. Namely, a set of arguments $S$ is $\beta$-conflict-free if the sum of the weights of attacks between arguments of $S$ does not exceed $\beta$. Details about defining acceptability semantics in WAF are given in [7].

In this paper, we do not use the inconsistency budget $\beta$ for defining the acceptability semantics and evaluating the set of arguments. However, we focus on the method developed in [5] where the authors defined some aggregation functions allowing to evaluate each extension for a given semantics (preferred for example). The method allows to compute the degree of global defense of each extension for a given semantics (preferred for example) on the basis of incoming, outgoing attacks and their weights.

Given a weighted argumentation framework WAF $=\langle\mathcal{A}, \mathcal{R}$, $w\rangle$, the corresponding standard argumentation framework denoted $W \hat{A} F=\langle\mathcal{A}, \mathcal{R}\rangle$ is obtained by removing the weights. Note that Definitions 5, 6 and 7 given in the following are from [5] where we consider the sum aggregation function. However, as cited in [5], other aggregation functions can be used such as leximax, maximin, etc. Before defining the best defended extensions, we firstly define the most attacking extensions and the least attacked extensions.

Definition 5 ( $\Sigma$-most attacking extensions): Let $\mathrm{WAF}=\langle\mathcal{A}, \mathcal{R}, w\rangle$ be a weighted argumentation framework. Let $\mathbb{E}$ be the set of extensions of $W \hat{A} F=$ $\langle\mathcal{A}, \mathcal{R}\rangle$ for a given semantics (preferred for example). Let $\Sigma$ be an aggregation function. For any extension $E$ of $\mathbb{E}$, one defines $\operatorname{Out}_{\Sigma}(E)=\Sigma_{\sigma \in E, \gamma \in \mathcal{A} \backslash E \text {, s.t. }(\sigma, \gamma) \in \mathcal{R}} w(\sigma, \gamma)$. The $\Sigma$-most attacking extensions of $\mathbb{E}$ are given by: $M a_{\Sigma}(\mathbb{E})=\operatorname{argmax}_{E \in \mathbb{E}}\left(O_{\Sigma} t_{\Sigma}(E)\right)$.

Definition 5 states that the $\Sigma$-most attacking extensions are those for which the sum of weights of outgoing attacks is maximal.

Definition 6 ( -least attacked extensions): Let WAF $=\langle\mathcal{A}, \mathcal{R}, w\rangle$ be a weighted argumentation framework. Let $\mathbb{E}$ be the set of extensions of $W \hat{A} F=\langle\mathcal{A}, \mathcal{R}\rangle$ for a given semantics. Let $\Sigma$ be an aggregation function. For any extension $E$ of $\mathbb{E}$, we define $\operatorname{In}_{\Sigma}(E)=\Sigma_{\sigma \in E, \gamma \in \mathcal{A} \backslash E \text {, s.t. }(\gamma, \sigma) \in \mathcal{R}} w(\gamma, \sigma)$. The $\Sigma$-least attacked extensions of $\mathbb{E}$ are given by: $L a_{\Sigma}(\mathbb{E})=\operatorname{argmin}_{E \in \mathbb{E}}\left(\operatorname{In}_{\Sigma}(E)\right)$.

Definition 6 states that the $\Sigma$-least attacked extensions are those having the smallest sum of weights of attacks towards them.

Definition 7 (Globaly $\Sigma$-best defended extensions): Let WAF $=\langle\mathcal{A}, \mathcal{R}, w\rangle$ be a weighted argumentation framework. Let $\mathbb{E}$ be the set of extensions of $W \hat{A} F=\langle\mathcal{A}, \mathcal{R}\rangle$ for a given semantics. Let $\Sigma$ be an aggregation function. For any extension $E$ of $\mathbb{E}$, the degree of its global defense is defined by: $\operatorname{De} f_{\Sigma}^{g}(E)=\operatorname{Out}_{\Sigma}(E)-\operatorname{In} n_{\Sigma}(E)$. The globally $\Sigma$-best defended extensions of $\mathbb{E}$ are given by : $G d b_{\Sigma}(\mathbb{E})=\operatorname{argmax}_{E \in \mathbb{E}}\left(\operatorname{De} f_{\Sigma}^{g}(\mathbb{E})\right)$. 
Example 3: Let us consider the weighted argumentation framework WAF $=\langle\mathcal{A}, \mathcal{R}, w\rangle$ given in Figure 3 where $\mathcal{A}=\left\{\sigma_{1}\right.$, $\left.\sigma_{2}, \sigma_{3}, \sigma_{4}\right\}, \mathcal{R}=\left\{\left(\sigma_{1}, \sigma_{2}\right),\left(\sigma_{2}, \sigma_{3}\right),\left(\sigma_{3}, \sigma_{4}\right),\left(\sigma_{4}, \sigma_{1}\right)\right\}$ and $w\left(\sigma_{1}, \sigma_{2}\right)=2, w\left(\sigma_{2}, \sigma_{3}\right)=5, w\left(\sigma_{3}, \sigma_{4}\right)=1, w\left(\sigma_{4}, \sigma_{1}\right)=7$.

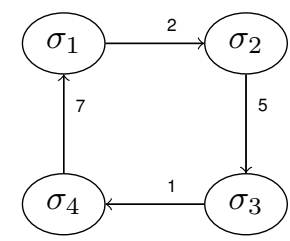

Figure 3. An example of WAF

The corresponding standard argumentation framework $W \hat{A} F=\langle\mathcal{A}, \mathcal{R}\rangle$ is obtained from $\mathrm{WAF}=\langle\mathcal{A}, \mathcal{R}, w\rangle$ by removing the weights. $W \hat{A} F$ has two preferred extensions $\mathbb{E}=\left\{E_{1}, E_{2}\right\}$ such that $E_{1}=\left\{\sigma_{1}, \sigma_{3}\right\}, E_{2}=\left\{\sigma_{2}, \sigma_{4}\right\}$.

From Definition 5, we have $\operatorname{Out}_{\Sigma}\left(E_{1}\right)=3, \operatorname{Out}_{\Sigma}\left(E_{2}\right)=12$. Thus, the $\Sigma$-most attacking extension of $\mathbb{E}$ is $E_{2}$. From Definition 6, we have $\operatorname{In}_{\Sigma}\left(E_{1}\right)=12, \operatorname{In}_{\Sigma}\left(E_{2}\right)=3$. Thus, the $\Sigma$-least attacked extension is $E_{2}$. From Definition 7 , we have $\operatorname{Def} f_{\Sigma}^{g}\left(E_{1}\right)=\operatorname{Out}_{\Sigma}\left(E_{1}\right)-\operatorname{In}_{\Sigma}\left(E_{1}\right)=-9$ and $\operatorname{Def}_{\Sigma}^{g}\left(E_{2}\right)=$ $\operatorname{Out}_{\Sigma}\left(E_{2}\right)-\operatorname{In}_{\Sigma}\left(E_{2}\right)=9$. Thus, the globally $\Sigma$-best defended extensions of $\mathbb{E}$ is $E_{2}$.

\section{TRAnSLATING COGNITIVE MAPS INTO WEIGHTED ARGUMENTATION FRAMEWORKS}

As mentioned previously, cognitive maps have the advantage to describe and capture the decision makers beliefs and judgments in a comprehensive manner. However, reasoning on this model presents some limits and remains a difficult task. For example, cognitive maps do not model uncertainty within the variables, and only deductive reasoning (predicting an effect given a cause) is possible in some situations, particularly when there are no circular relations. Thus, we propose to represent the decision maker's judgments and beliefs in the form of arguments and attacks between them. Namely, the aim is to start from the constructed cognitive map and build the corresponding weighted argumentation framework. The choice of WAF is justified by the fact that in WAF, weights are assigned to attacks. In cognitive map, each influence relation is associated with a numerical value indicating the strength of the relation. Thus, the link between the two formalisms can be naturally established.

To translate a constructed cognitive map into weighted argumentation framework, we have to firstly define an argument. As defined in different works [2], an argument is a pair where the first element is a set of premises, the second element is a claim, and from the premises a claim is inferred. Given a cognitive map, the arguments of its corresponding WAF are structured from the influences between the concepts of the $C M$. The premise of an argument corresponds to the possible state of the causal concept and its claim corresponds to the possible state of the effect concept. We use $\operatorname{pr}(\sigma)$ (resp. $c l(\sigma))$ to denote the premise (resp. claim) of the argument $\sigma$.

Definition 8 (Arguments): Let $C M=\langle\mathcal{C}, \mathcal{I}, \mathrm{V}$, val $\rangle$ be a cognitive map and $\mathcal{C}$ be a set of its concepts. Let $c_{1}$, $c_{2}$ be two concepts in $\mathcal{C}$ such that $\operatorname{State}\left(c_{1}\right)=\left\{c_{1}^{-}, c_{1}^{+}\right\}$, $\operatorname{State}\left(c_{2}\right)=\left\{c_{2}^{-}, c_{2}^{+}\right\}$and $I \in \mathcal{I}$ be an influence between $c_{1}$ and $c_{2}$. An argument is defined over an influence $I$ denoted by $\sigma$ : State $\left(c_{1}\right) \rightarrow \operatorname{State}\left(c_{2}\right)$ such that $\operatorname{State}\left(c_{1}\right)$ is either $c_{1}^{-}$ or $c_{1}^{+}$and $\operatorname{State}\left(c_{2}\right)$ is either $c_{2}^{-}$or $c_{2}^{+}$according to Property 1 .

Assume that we have an argument $\sigma$ such that $\sigma$ : $\operatorname{State}\left(c_{1}\right) \rightarrow \operatorname{State}\left(c_{2}\right)$. Let State $\left(c_{1}\right)=c_{1}^{-}$and $\operatorname{State}\left(c_{2}\right)=$ $c_{2}^{+}$then $\sigma: c_{1}^{-} \rightarrow c_{2}^{+}$can be read as " $c_{1}^{-}$entails $c_{2}^{+}$" or " $c_{1}^{-}$ causes $c_{2}^{+} . \operatorname{pr}(\sigma)=c_{1}^{-}, \operatorname{cl}(\sigma)=c_{2}^{+}$. Note that an argument has one premise and one claim in the obtained WAF.

Proposition 1: Let $C M=\langle\mathcal{C}, \mathcal{I}, \mathrm{V}$, val $\rangle$ be a cognitive map. $\forall I \in \mathcal{I}$, it holds that there are exactly two arguments $\sigma, \sigma^{\prime}$ that can be defined over $I$.

Proof: Let $C M=\langle\mathcal{C}, \mathcal{I}, \mathrm{V}$, val $\rangle$ be a cognitive map and $\mathcal{C}$ be a set of its concepts. Let $c_{1}, c_{2}$ be two concepts in $\mathcal{C}$ such that State $\left(c_{1}\right)=\left\{c_{1}^{-}, c_{1}^{+}\right\}, \operatorname{State}\left(c_{2}\right)=\left\{c_{2}^{-}, c_{2}^{+}\right\}$and $I \in \mathcal{I}$ be an influence between $c_{1}$ and $c_{2}$. From Definition 8 , an argument is defined over an influence $I$ denoted by $\sigma$ : State $\left(c_{1}\right) \rightarrow \operatorname{State}\left(c_{2}\right)$. We have $\operatorname{State}\left(c_{1}\right)=\left\{c_{1}^{-}, c_{1}^{+}\right\}$and State $\left(c_{2}\right)=\left\{c_{2}^{-}, c_{2}^{-}\right\}$. Thus, the following arguments can be defined from $I$ :

- $\sigma_{1}: c_{1}^{-} \rightarrow c_{2}^{+}$or $\sigma_{2}: c_{1}^{-} \rightarrow c_{2}^{-}$, and

- $\sigma_{3}: c_{1}^{+} \rightarrow c_{2}^{+}$or $\sigma_{4}: c_{1}^{+} \rightarrow c_{2}^{-}$.

However, if we have $c_{1}^{-}$(namely, decreasing of $c_{1}$ ), then only one case holds concerning $c_{2}$. Namely, we have either $c_{2}^{+}$or $c_{2}^{-}$ (see Property 1). The same observation is available if we have $c_{1}^{+}$. Thus, only two arguments are defined over each influence $I$. More precisely, we have either $\sigma_{1}$ and $\sigma_{4}$ or $\sigma_{2}$ and $\sigma_{3}$.

Example 4: From the cognitive map of Figure 1, the following arguments are constructed:

\begin{tabular}{ll}
\hline$\sigma_{1}: G M^{-} \rightarrow W T^{-}$ & $\sigma_{2}: G M^{+} \rightarrow W T^{+}$ \\
$\sigma_{3}: W T^{-} \rightarrow F A^{+}$ & $\sigma_{4}: W T^{+} \rightarrow F A^{-}$ \\
$\sigma_{5}: F A^{-} \rightarrow F S^{+}$ & $\sigma_{6}: F A^{+} \rightarrow F S^{-}$ \\
$\sigma_{7}: G M^{-} \rightarrow M^{-}$ & $\sigma_{8}: G M^{+} \rightarrow M^{+}$ \\
$\sigma_{9}: M M^{-} \rightarrow F S^{+}$ & $\sigma_{10}: M^{+} \rightarrow F S^{-}$ \\
\hline
\end{tabular}

The argument $\sigma_{1}$ is defined over the influence between the concept $G M$ and the concept $W T$ (see Figure 1). $\operatorname{pr}\left(\sigma_{1}\right)=$ $G M^{-}$and $\operatorname{cl}\left(\sigma_{1}\right)=W T^{-}$. It means that a decrease of gold mining will cause (or entails) a decrease of water turbidity. The argument $\sigma_{3}$ is defined over the influence between the concept $W T$ and the concept $F A$ (see Figure 1). $\operatorname{pr}\left(\sigma_{3}\right)=$ $W T^{-}$and $\operatorname{cl}\left(\sigma_{3}\right)=F A^{+}$. It means that a decrease of water turbidity will cause (or entails) an increase of fishing activity.

The knowledge represented in a cognitive map contains positive and negative influence relations which are contradictory. Namely, some concepts might cause increasing a given concept $c$ and other might cause decreasing of $c$. Thus, we cannot have increase and decrease of the same concept at 
the same time (if such result holds, we have an inconsistent situation). In argumentation theory, inconsistency due to conflicting views or contradictory conclusions is represented by attack relations between arguments. In our problem, we distinguish two types of attack relations between arguments. The first one occurs between two arguments contradicting each other because their claims are mutually exclusive. The second one occurs when some arguments challenge the inference of another argument. The former are called rebuttal attacks while the later are called undercut attacks. Let us use the complement notation to express contradictory or inconsistent elements such as $\operatorname{cl}(\sigma)=\overline{c l\left(\sigma^{\prime}\right)}$ that expresses the claims of the arguments $\sigma$ and $\sigma^{\prime}$ are contradictory.

Definition 9 (Rebuttal attack): Given two arguments $\sigma$ and $\sigma^{\prime}$, we say that $\sigma$ attacks $\sigma^{\prime}$ and the attack is said to be rebuttal iff $\operatorname{cl}(\sigma)=\overline{c l}\left(\sigma^{\prime}\right)$. The set of rebuttal attacks is denoted by $\mathcal{R}_{r}$.

Property 2: Let $\sigma, \sigma^{\prime}$ be two arguments in $\mathcal{A}$ such that $\operatorname{cl}(\sigma)=\overline{c l}\left(\sigma^{\prime}\right)$. It holds that we have $\sigma$ attacks $\sigma^{\prime}$ and $\sigma^{\prime}$ attacks $\sigma$.

Proof: Let $\sigma, \sigma^{\prime}$ be two arguments in $\mathcal{A}$. By Definition, the rebuttal attack is symmetric. Thus, if we have $\operatorname{cl}(\sigma)=$ $\overline{\operatorname{cl}\left(\sigma^{\prime}\right)}$, then we have $\mathcal{R}_{r}=\left\{\left(\sigma, \sigma^{\prime}\right),\left(\sigma^{\prime}, \sigma\right)\right\}$.

Definition 10 (Undercut attack): Given an argument $\sigma \in$ $\mathcal{A}$, if $\exists \sigma^{\prime} \in \mathcal{A}$ such that $\operatorname{pr}\left(\sigma^{\prime}\right)=\overline{c l}(\sigma)$ then $\sigma$ undercuts $\sigma^{\prime}$. The undercut attack is denoted by $\mathcal{R}_{u}$ and $\mathcal{R}_{u}=\left\{\left(\sigma, \sigma^{\prime}\right)\right\}$.

Property 3: Let $\sigma, \sigma^{\prime}$ be two arguments in $\mathcal{A}$ such that $\operatorname{pr}\left(\sigma^{\prime}\right)=\overline{c l}(\sigma)$. The attack between $\sigma$ and $\sigma^{\prime}$ is asymmetric. Proof: Let $\sigma, \sigma^{\prime}$ be two arguments in $\mathcal{A}$ and assume that $\mathcal{R}_{u}=\left\{\left(\sigma, \sigma^{\prime}\right)\right\}$. By Definition, this means that $\sigma^{\prime}$ is no longer justified because of $\sigma$. Thus, we cannot have $\sigma^{\prime}$ that attacks $\sigma$.

Example 5: In Example 4, 10 arguments are defined from the cognitive map of Figure 1.

- $\mathcal{R}_{r}=\left\{\left(\sigma_{1}, \sigma_{2}\right),\left(\sigma_{2}, \sigma_{1}\right),\left(\sigma_{3}, \sigma_{4}\right),\left(\sigma_{4}, \sigma_{3}\right),\left(\sigma_{5}, \sigma_{6}\right)\right.$, $\left(\sigma_{6}, \sigma_{5}\right),\left(\sigma_{7}, \sigma_{8}\right),\left(\sigma_{8}, \sigma_{7}\right),\left(\sigma_{5}, \sigma_{10}\right),\left(\sigma_{10}, \sigma_{5}\right),\left(\sigma_{6}, \sigma_{9}\right)$, $\left.\left(\sigma_{9}, \sigma_{6}\right),\left(\sigma_{9}, \sigma_{10}\right),\left(\sigma_{10}, \sigma_{9}\right)\right\}$.

- $\mathcal{R}_{u}=\left\{\left(\sigma_{2}, \sigma_{3}\right),\left(\sigma_{1}, \sigma_{4}\right),\left(\sigma_{3}, \sigma_{5}\right),\left(\sigma_{4}, \sigma_{6}\right),\left(\sigma_{7}, \sigma_{10}\right)\right.$, $\left.\left(\sigma_{8}, \sigma_{9}\right)\right\}$.

In weighted argumentation framework, each attack is associated with a weight, indicating the relative strength of that attack. In [7], the authors discussed where weights come from: from votes in multi-agent systems, or as measures of inconsistency between pairs of arguments, etc.

In the weighted argumentation framework obtained from a given cognitive map, the weights correspond to the values associated with influences between concepts of the cognitive map.

Definition 11 (Attack weight): Let $C M=\langle\mathcal{C}, \mathcal{I}$, V, val $\rangle$ be a cognitive map, $I \in \mathcal{I}$ such that $\operatorname{val}(I)=\left[v, v^{\prime}\right]$ and WAF $=$
$\langle\mathcal{A}, \mathcal{R}, w\rangle$ be its corresponding WAF where $\mathcal{A}$ is the set of arguments, $\mathcal{R}=\mathcal{R}_{r} \cup \mathcal{R}_{u}$ is the set of rebuttal and undercuting attacks and $w$ is a function that maps a natural number to each attack.

Let $\left(\sigma, \sigma^{\prime}\right),\left(\sigma^{\prime}, \sigma\right) \in \mathcal{R}_{r}$.

- If $\operatorname{pr}(\sigma)=c_{1}^{-}$then $w\left(\sigma, \sigma^{\prime}\right)=|v|$ and $w\left(\sigma^{\prime}, \sigma\right)=\left|v^{\prime}\right|$.

- If $\operatorname{pr}(\sigma)=c_{1}^{+}$then $w\left(\sigma, \sigma^{\prime}\right)=\left|v^{\prime}\right|$ and $w\left(\sigma^{\prime}, \sigma\right)=|v|$. Let $\left(\sigma, \sigma^{\prime}\right) \in \mathcal{R}_{u}$.

- If $\operatorname{pr}(\sigma)=c_{1}^{-}$then $w\left(\sigma, \sigma^{\prime}\right)=|v|$.

- If $\operatorname{pr}(\sigma)=c_{1}^{+}$then $w\left(\sigma, \sigma^{\prime}\right)=\left|v^{\prime}\right|$.

Example 6: In Figure 8, we have an example of an influence relation extracted from the cognitive map given in Figure 1 and the corresponing arguments and the attack relations between them.

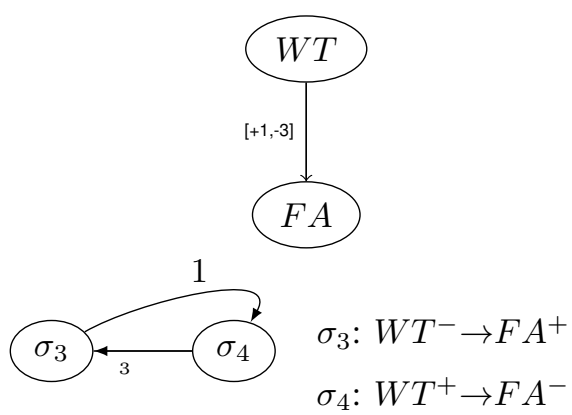

Figure 4. The corresponding arguments and attack relations obtained from the influence between two concepts.

In Figure 8, the value $v=+1$ of the influence between $W T$ and $F A$ represents the fact that a decrease of $W T$ causes a small increase of $F A$. So, the defined argument (Figure 8) is $\sigma_{3}: W T^{-} \rightarrow F A^{+}$. The value $v^{\prime}=-3$ of the influence between $W T$ and $F A$ expresses that an increase of $W T$ causes a high decrease of $F A$. So, the defined argument is $\sigma_{4}: W T^{+} \rightarrow F A^{-}$. From Definition 9, we have $\sigma_{3}$ and $\sigma_{4}$ attack each other and from Definition 11, we have $w\left(\sigma_{3}\right.$, $\left.\sigma_{4}\right)=1$ and $w\left(\sigma_{4}, \sigma_{3}\right)=3$. We can observe that the weights assigned to the attacks are not negative. This is explained by the fact that the negative or positive sign of the weight is represented through the claim of an argument (increasing (resp. decreasing) if the value of the influence from which the argument is constructed is positive (resp. negative)). There is no interest to have negative weights because, in cognitive map, the values -1 and +1 represent low influence, -2 and +2 represent medium influence, -3 and +3 represent high influence. Thus, in $W A F$, the attack which is qualified to be low (resp. medium, high) is represented by the weight 1 (resp. 2, 3). So, $w\left(\sigma_{3}, \sigma_{4}\right)=1$ means that the attack from $\sigma_{3}$ on $\sigma_{4}$ is qualified to be low, while $w\left(\sigma_{4}, \sigma_{3}\right)=3$ means that the attack from $\sigma_{4}$ on $\sigma_{3}$ is qualified to be high. More precisely, since the attack from $\sigma_{4}$ to $\sigma_{3}$ is stronger than the one from $\sigma_{3}$ to $\sigma_{4}$, then a decrease in fishing activity (which is a claim of $\sigma_{4}$ ) is the more plausible situation than its increase (which is a claim of $\sigma_{3}$ ).

Definition 12: Let $C M=\langle\mathcal{C}, \mathcal{I}$, V, val $\rangle$ be a cognitive map where $\mathcal{C}$ is a set of concepts, $\mathcal{I}$ is a set of influences between 
concepts, val is a function that assigns two values to each influence. A weighted argumentation framework built from $C M$ is a triple WAF $=\langle\mathcal{A}, \mathcal{R}, w\rangle$ where: $\mathcal{A}$ is a set of arguments defined from $\mathcal{C}$ using Definition $8, \mathcal{R}$ is a set of attacks between arguments defined using Definition 9 for rebuttal attacks and Definition 10 for undercut attacks and $w$ is a function that assigns a natural number to each attack defined using Definition 11.

Example 7: Let us consider the cognitive map given in Example 1. The weighted argumentation framework built from this later is given in the Figure 5. The bidirectional arcs represent rebuttal attacks while the unidirectional arcs represent undercut attacks.

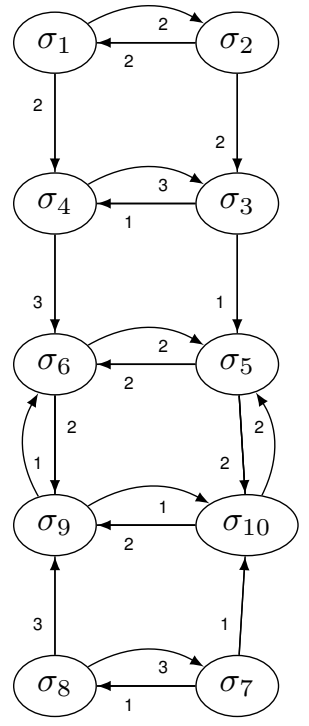

Figure 5. The WAF associated to the cognitive map

The arguments are the following:

\begin{tabular}{ll}
\hline$\sigma_{1}: G M^{-} \rightarrow W T^{-}$ & $\sigma_{2}: G M^{+} \rightarrow W T^{+}$ \\
$\sigma_{3}: W T^{-} \rightarrow F A^{+}$ & $\sigma_{4}: W T^{+} \rightarrow F A^{-}$ \\
$\sigma_{5}: F A^{-} \rightarrow F S^{+}$ & $\sigma_{6}: F A^{+} \rightarrow F S^{-}$ \\
$\sigma_{7}: G M^{-} \rightarrow M^{-}$ & $\sigma_{8}: G M^{+} \rightarrow M^{+}$ \\
$\sigma_{9}: M^{-} \rightarrow F S^{+}$ & $\sigma_{10}: M^{+} \rightarrow F S^{-}$ \\
\hline
\end{tabular}

\section{REASONING IN THE OBTAINED WAF}

In argumentation theory, it is usually assumed that premises of arguments always hold. However, for real applications such as the problem of fishing activity studied in this paper, it is possible that only some events are observed. For example, an increase of gold mining and a decrease of water turbidity. Thus, for more efficient reasoning in the obtained WAF from a given cognitive map, we propose to enrich it for dealing with dynamics through the consideration of a varying set of observations. In the following, we introduce a notion of observations, we will assume that it is a set of consistent elements representing the current state of the world such as a decrease of fishing activity. Thus, we must define what we call active arguments and active attacks.
An argument is active because its premise belongs to the set of observations as stated by Item 1 of Definition 15 given below. However, an argument can be active by other active arguments and not observations what will directly activating it. Namely, an argument can also be activated by what we call i) its supporting argument (defined in Definition 13 given below) which may be also active or by ii) its undercutting argument (defined in Definition 14 given below) which may be also active.

Definition 13 (Supporting argument): Let $C M$ be a cognitive map and $\mathrm{WAF}=\langle\mathcal{A}, \mathcal{R}, w\rangle$ be its corresponding weighted argumentation framework. Let $\sigma, \sigma^{\prime}$ be two arguments in $\mathcal{A}$. We say that $\sigma$ is a supporting argument of $\sigma^{\prime}$ iff $\operatorname{pr}\left(\sigma^{\prime}\right)=\operatorname{cl}(\sigma)$.

Definition 14 (Undercutting argument): Let $C M$ be a cognitive map and $\mathrm{WAF}=\langle\mathcal{A}, \mathcal{R}, w\rangle$ be its corresponding weighted argumentation framework. Let $\sigma, \sigma^{\prime}$ be two arguments in $\mathcal{A}$. We say that $\sigma$ is an undercutting argument of $\sigma^{\prime}$ iff $\operatorname{pr}\left(\sigma^{\prime}\right)=\overline{c l}(\sigma)$.

Definition 15 (Active argument): Let $C M$ be a cognitive map and $\mathrm{WAF}=\langle\mathcal{A}, \mathcal{R}, w\rangle$ be its corresponding weighted argumentation framework. Let $\mathcal{O}$ be a set of observations. An argument $\sigma \in \mathcal{A}$ is active with respect to $\mathcal{O}$ iff:

- $\operatorname{pr}(\sigma) \in \mathcal{O}$, or

- $\exists \sigma^{\prime} \in \mathcal{A}$ which is an active and supporting argument of $\sigma$, or

- $\exists \sigma^{\prime} \in \mathcal{A}$ which is an active and undercutting argument of $\sigma$.

$\mathcal{A}_{A c t} \subseteq \mathcal{A}$ denotes the set of active arguments.

Example 8: Let us consider the arguments of Example 5 and assume that the set of observations $\mathcal{O}=\left\{G M^{+}\right\}$then $\mathcal{A}_{A c t}$ $=\left\{\sigma_{2}, \sigma_{3}, \sigma_{4}, \sigma_{5}, \sigma_{6}, \sigma_{8} \sigma_{9}, \sigma_{10}\right\} . \sigma_{2}$ (resp. $\sigma_{8}$ ) is active directly from $\mathcal{O}$ since we have $\operatorname{pr}\left(\sigma_{2}\right)$ (resp. $\left.\operatorname{pr}\left(\sigma_{8}\right)\right) \in \mathcal{O}$. $\sigma_{4}$ is activated by $\sigma_{2}$ since we have $\operatorname{pr}\left(\sigma_{4}\right)=\operatorname{cl}\left(\sigma_{2}\right)$ (from Definition 13, $\sigma_{2}$ is active and it is a supporting argument of $\left.\sigma_{4}\right) . \sigma_{5}$ is activated by $\sigma_{4}\left(\sigma_{4}\right.$ is a supporting argument of $\left.\sigma_{5}\right)$. $\sigma_{6}$ is activated by $\sigma_{4}\left(\sigma_{4}\right.$ is active and it is an undercutting argument of $\sigma_{6}$ ), etc.

As seen above, there exists rebuttal and undercut attacks. Thus, a rebuttal attack is active if and only if both the attacker and the attacked are in the set of active arguments. An undercut attack is active if and only if the attacker argument is the set of active arguments.

Definition 16 (Active rebuttal attacks): The set of active rebuttal attacks is a subset $\mathcal{R}_{r}^{A c t} \subseteq \mathcal{R}$ such that $\left(\sigma, \sigma^{\prime}\right) \in$ $\mathcal{R}_{r}^{A c t}$ iff $\sigma, \sigma^{\prime} \in \mathcal{A}_{A c t}$.

Definition 16 states that a rebuttal attack is active if and only if both the attacker and the attacked arguments are in the set of active arguments.

Definition 17 (Active undercut attacks): The set of active undercuts attacks is a subset $\mathcal{R}_{u}^{A c t} \subseteq \mathcal{R}$ such that $\left(\sigma, \sigma^{\prime}\right) \in$ $\mathcal{R}_{u}^{A c t}$ iff $\sigma \in \mathcal{A}_{\text {Act }}$.

We can observe that in Definition 17, an undercut attack is active if and only if the attacker argument is active. This can be explained by the fact that by Definition 14, if the 
undercutting argument is active, then it activates the argument which attacks.

Definition 18 (Active weights): Let $\sigma, \sigma^{\prime}$ be two arguments in $\mathcal{A}_{A c t}, w\left(\sigma, \sigma^{\prime}\right)$ is active iff $\left(\sigma, \sigma^{\prime}\right) \in \mathcal{R}_{r}^{A c t}$ or $\left(\sigma, \sigma^{\prime}\right)$ $\in \mathcal{R}_{u}^{A c t}$. We use $w_{A c t}$ to denote a function that assigns to each active attack its associated weight.

Definition 18 states that if a given attack (rebuttal or undercut) between two arguments is active then the weight associated to this attack is also active.

Once the WAF is built, we have to define acceptability semantics such as preferred extensions. The advantage of WAF compared to Dung abstract argumentation framework is the possibility to consider the available weights in order to select the extensions which best defend themselves. In Subsection III-B, we give some definitions allowing to compute the degree of global defense of each extension. In addition, once the best defended extensions are determined, it is possible to give explanation of the results on the basis of the arguments that are in the best extensions (see the following example).

Example 9: Let us consider the cognitive map given in Example 1. The weighted argumentation framework built from this later is given in the Figure 5. In the following we consider two observations:

1) Case 1: Assume that we have the set of observations $\mathcal{O}_{1}=\left\{G M^{+}\right\}$.

- From Definitions $15, \mathcal{A}_{A c t}=\left\{\sigma_{2}, \sigma_{3}, \sigma_{4}, \sigma_{5}, \sigma_{6}\right.$, $\left.\sigma_{8}, \sigma_{9}, \sigma_{10}\right\}$.

- From Definition $16, \mathcal{R}_{r}^{A c t}=\left\{\left(\sigma_{3}, \sigma_{4}\right),\left(\sigma_{4}, \sigma_{3}\right),\left(\sigma_{5}\right.\right.$, $\left.\left.\sigma_{6}\right),\left(\sigma_{6}, \sigma_{5}\right),\left(\sigma_{9}, \sigma_{10}\right),\left(\sigma_{10}, \sigma_{9}\right)\right\}$.

- From Definition $17, \mathcal{R}_{u}^{A c t}=\left\{\left(\sigma_{2}, \sigma_{3}\right),\left(\sigma_{3}, \sigma_{5}\right),\left(\sigma_{4}\right.\right.$, $\left.\left.\sigma_{6}\right),\left(\sigma_{8}, \sigma_{9}\right)\right\}$.

- From Definition 18, each active rebuttal and undercut attack is associated with its active weight.

The weighted argumentation framework obtained from $\mathcal{O}_{1}$ is a sub-WAF $=\left\langle\mathcal{A}_{A c t}, \mathcal{R}_{r}^{A c t} \cup \mathcal{R}_{u}^{A c t}, w_{A c t}\right\rangle$ given in Figure 6.

The standard sub-argumentation framework sub-WAF corresponding to the sub-WAF of Figure 6 has two preferred extensions: $E_{1}=\left\{\sigma_{2}, \sigma_{6}, \sigma_{8}, \sigma_{10}\right\}, E_{2}=\left\{\sigma_{2}\right.$, $\left.\sigma_{4}, \sigma_{5}, \sigma_{8}\right\}$.

From Definition 5, we have $\operatorname{Out}\left(E_{1}\right)$ $=w\left(\sigma_{2}, \sigma_{3}\right)+w\left(\sigma_{6}, \sigma_{5}\right)+w\left(\sigma_{6}, \sigma_{9}\right)+w\left(\sigma_{8}, \sigma_{9}\right)+w\left(\sigma_{10}, \sigma_{9}\right)$ $+w\left(\sigma_{10}, \sigma_{5}\right)=13 . \quad \operatorname{Out}\left(E_{2}\right)=w\left(\sigma_{2}, \sigma_{3}\right)+w\left(\sigma_{4}, \sigma_{6}\right)+$ $w\left(\sigma_{4}, \sigma_{3}\right)+w\left(\sigma_{5}, \sigma_{6}\right)+w\left(\sigma_{5}, \sigma_{10}\right)+w\left(\sigma_{8}, \sigma_{9}\right)=15$. Thus, $\Sigma$-most attacking extension is $E_{2}$.

Using Definition 6, $\operatorname{In}\left(E_{1}\right)=9 . \quad \operatorname{In}\left(E_{2}\right)=6 . \quad$ Thus, $\Sigma$-least attacked extension is $E_{2}$. Using Definition 7 , the globally $\Sigma$-best defended extension is $E_{2}$. This means that the most plausible situation regarding the judgments represented in the above cognitive map and the given observations concerns the increasing of fish stock $\left(F S^{+}\right)$which is the claim of $\sigma_{5}$ since it belongs to the $\Sigma$-best defended extension. The result is explained (i.e. increasing of fish stock) by (i) an

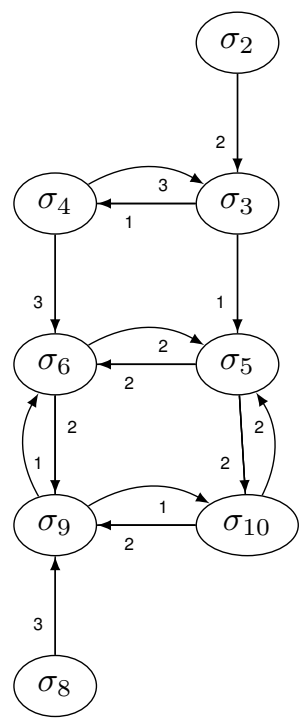

Figure 6. The sub-WAF associated to the observation $\mathcal{O}_{1}$

increase of water turbidity $\left(W T^{+}\right)$which is the claim of $\sigma_{2}$ s.t. $\sigma_{2}$ belongs to the $\Sigma$-best defended extension, (ii) a decrease of fishing activity $\left(F A^{-}\right)$, (iii) an increase of mercury $\left(M^{+}\right) . W T^{+}$and $M^{+}$are explained by an increase of gold mining $\left(G M^{+}\right.$which is in the set of observations), $F A^{-}$is explaind by $W T^{+}$.

2) Case 2: Assume that we have the set of observations $\mathcal{O}_{2}=\left\{W T^{-}, M^{-}\right\}$

- From Definitions $15, \mathcal{A}_{A c t}=\left\{\sigma_{3}, \sigma_{5}, \sigma_{6}, \sigma_{9}\right\}$.

- From Definition $16, \mathcal{R}_{r}^{A c t}=\left\{\left(\sigma_{5}, \sigma_{6}\right),\left(\sigma_{6}, \sigma_{5}\right),\left(\sigma_{9}\right.\right.$, $\left.\left.\sigma_{6}\right),\left(\sigma_{6}, \sigma_{9}\right)\right\}$.

- From Definition 17, $\mathcal{R}_{u}^{A c t}=\left\{\left(\sigma_{3}, \sigma_{5}\right)\right\}$.

- From Definition 18, each active rebuttal and undercut attack is associated with its weight.

The weighted argumentation framework obtained from $\mathcal{O}_{2}$ is a sub-WAF $=\left\langle\mathcal{A}_{A c t}, \mathcal{R}_{r}^{A c t} \cup \mathcal{R}_{u}^{A c t}, w_{A c t}\right\rangle$ given in Figure 7.

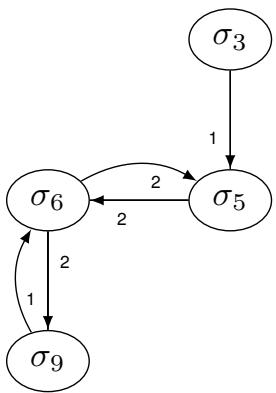

Figure 7. The sub-WAF associated to the observation $\mathcal{O}_{2}$

The standard sub-argumentation framework sub-WAF corresponding to the sub-WAF of Figure 7 has two preferred extensions: $E_{1}=\left\{\sigma_{3}, \sigma_{6}\right\}, E_{2}=\left\{\sigma_{3}, \sigma_{9}\right\}$.

- Using Definition 5, Out $t_{\Sigma}\left(E_{1}\right)=5$. Out $t_{\Sigma}\left(E_{2}\right)=2$. Thus, $\Sigma$-most attacking extension is $E_{1}$.

- Using Definition 6, $\operatorname{In}_{\Sigma}\left(E_{1}\right)=3 . \operatorname{In}_{\Sigma}\left(E_{2}\right)=2$. Thus, 
$\Sigma$-least attacked extension is $E_{2}$.

- Using Definition 7, the degree of the defense of $E_{1}$ is given as follows (resp. $\left.E_{2}\right)$ : $\operatorname{Def} f_{\Sigma}^{g}\left(E_{1}\right)=$ $\operatorname{Out}_{\Sigma}\left(E_{1}\right)-\operatorname{In}_{\Sigma}\left(E_{1}\right)=2$.

$\operatorname{Def} f_{\Sigma}^{g}\left(E_{2}\right)=\operatorname{Out}_{\Sigma}\left(E_{2}\right)-\operatorname{In}_{\Sigma}\left(E_{2}\right)=0$.

Thus the globally $\Sigma$-best defended extension is $E_{1}$. This means that the most plausible situation regarding the judgments represented in the above cognitive map and the given observations concerns decreasing of fish stock $\left(F S^{-}\right)$which is the claim of $\sigma_{6}$ since it belongs to the $\Sigma$-best defended extension. The result is explained (i.e. decreasing of fish stock) by an increase of fishing activity $\left(F A^{+}\right)$which is the claim of $\sigma_{3}$ s.t. $\sigma_{3}$ belongs to the $\Sigma$-best defended extension. $F A^{+}$is explained by $W T^{-}$(WT $T^{-}$is in the set of observations).

Note that if there are circular relations in the cognitive map, the corresponding WAF can be obtained in the same way and reasoning about it can be applied following the method described above. In the following Figure, we have an example of circular influence relation and the corresponding WAF.

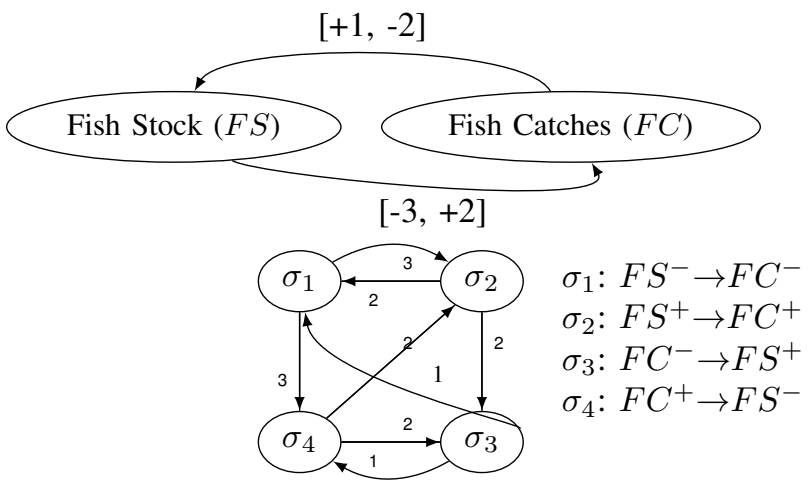

Figure 8. An example of the WAF obtained from two concepts with circular influence relation.

\section{CONCLUSION}

The contribution of this paper is about two formalisms for knowledge representation and reasoning. The first one is cognitive map which is graphical model allowing to easily organize individuals' judgments about a given problem. The second one is weighted argumentation framework which extends Dung $A F$ by associating a weight to each attack between arguments. However, even if cognitive maps are easier to grasp and close to the way in which knowledge is represented by human beings, reasoning about the represented knowledge has some limits.

Our proposition consists to transform any cognitive map in its corresponding weighted argumentation framework. Reasoning with WAF is very interesting, especially in situations of inconsistency induced in case when there are many cognitive maps. In addition, we proposed a method that allows us to deal with dynamics through the consideration of a varying set of observations in the obtained WAF. For example, having an observation about an increase in the number of fishers in a fishing activity problem, only some arguments and attacks can be active. Works dealing with transformations from cognitive maps into other formalisms exist such as those proposed in [10], [11], [14]. In particular, in these works, the cognitive map is either integrated or transformed into Bayesian network. However, to the best of our knowledge, our approach is the first proposing transformation into argumentation framework. Reasoning in the obtained WAF seems very interesting. So, as future work, we would study others aspects of this point. In particular, reasoning with uncertain observations, explanation of results on the basis of objective or subjective acceptance of arguments and abductive WAF in the spirit of the approach developed in [3]. In addition, in this paper, we considered only one cognitive map. However it is possible to have many cognitive maps since many fishers having different and conflicting judgments and viewpoints participate in constructing the cognitive map. Thus, our aim in future work is to deal with this problem.

\section{REFERENCES}

[1] Salem Benferhat, Amélie Levray, and Karim Tabia. On the analysis of probability-possibility transformations: Changing operations and graphical models. In In 13th European ConferenceSymbolic and Quantitative Approaches to Reasoning with Uncertainty ECSQARU, 2015.

[2] Philippe Besnard and Anthony Hunter. Constructing argument graphs with deductive arguments: a tutorial. Argument \& Computation, 5(1):530, 2014.

[3] Richard Booth, Dov M. Gabbay, Souhila Kaci, Tjitze Rienstra, and Leendert W. N. van der Torre. Abduction and dialogical proof in argumentation and logic programming. In ECAI 2014 - 21st European Conference on Artificial Intelligence, pages 117-122, 2014.

[4] Brahim Chaib-draa. Causal maps : Theory, implementation and practical applications in multiagent environments. In IEEE Transaction On Knowledge and Data Engineering, 2001.

[5] Sylvie Coste-Marquis, Sébastien Konieczny, Pierre Marquis, and Mohand Akli Ouali. Selecting extensions in weighted argumentation frameworks. In Computational Models of Argument, pages 342-349, 2012.

[6] Phan Minh Dung. On the acceptability of arguments and its fundamental role in non-monotonic reasoning, logic programming and $\mathrm{n}$-person games. Artificial Intelligence, 77(2):321-358, 1995.

[7] Paul E. Dunne, Anthony Hunter, Peter McBurney, Simon Parsons, and Michael Wooldridge. Weighted argument systems: Basic definitions, algorithms, and complexity results. Artificial Intelligence., 175(2):457486, 2011.

[8] Colin Eden and Steve Cropper. Cognitive mapping - a user's guide. In Working paper, February 1990.

[9] Bei Shui Liao, Li Jin, and Robert C. Koons. Dynamics of argumentation systems: A division-based method. Artif. Intell., 175(11):1790-1814, 2011.

[10] Sucheta Nadkarni and Prakash P Shenoy. A bayesian network approach to making inferences in causal maps. European Journal of Operational Research, 128:479-498, 2001.

[11] E. Romano R. Giordano, E. Preziosi. An integration between cognitive map and bayesian belief network for conflicts analysis in drought management. In International Environmental Modelling and Software Society (iEMSs), pages 60-65, 2010.

[12] Nicolás D. Rotstein, Martín O. Moguillansky, Alejandro Javier García, and Guillermo Ricardo Simari. A dynamic argumentation framework. In Computational Models of Argument, pages 427-438, 2010.

[13] Chiaki Sakama. Abduction in argumentation frameworks and its use in debate games. In New Frontiers in Artificial Intelligence - JSAI-isAI 2013 Workshops, pages 285-303, 2013.

[14] Karima Sedki and Louis Bonneau de Beaufort. Cognitive maps for knowledge represenation and reasoning. In IEEE 24th International Conference on Tools with Artificial Intelligence, ICTAI 2012, Athens, Greece, November 7-9, 2012, pages 1035-1040, 2012.

[15] Lotfi A. Zadeh. Fuzzy sets as a basis for a theory of possibility. Fuzzy Sets and Systems, 100:9-34, 1999. 